\title{
LA ENSEÑANZA DE LA HISTORIA EN ITALIA, ENTRE PASADO, REFORMAS Y HORIZONTES FUTUROS ${ }^{1}$
}

The Teaching of History in Italy, between Past, Reforms and Future Horizons

\author{
Beatrice Borghi \\ b.borghi@unibo.it \\ Università di Bologna. Italia \\ Debora Montanari \\ debora.montanari5@studio.unibo.it \\ Università di Bologna. Italia
}

Fecha de recepción: 13/04/2021

Fecha de aceptación: 14/05/2021

Resumen: El artículo expone los ejemplos más sobresalientes de las etapas que han caracterizado las reformas de la enseñanza en Italia, desde los currículos educativos del Ochocientos hasta las recientes normativas nacionales en materia de educación cívica y del patrimonio. En los últimos veinte años se han llevado a cabo diferentes reformas legislativas (Moratti 2003, Gelmini 2008, Buona Scuola 2015), cada una de las cuales introdujo significativos cambios, que todavía continúan vigentes en los programas curriculares. Veremos una descripción general desde la eliminación del examen estatal en el quinto año de la Escuela Primaria, la alternancia de prácticas obligatorias en las secundarias de II grado y la formación obligatoria y permanente de los docentes en activo; hasta la reciente aprobación en 2019 de la Ley sobre la educación cívica, como tema independiente, aunque relacionado con los programas de orden y grado, cuya introducción tuvo lugar en el curso 2020/2021. En las conclusiones se ofrecen unas orientaciones para la mejora de las prácticas didácticas llevadas a cabo en estas

1 Traducción de Juan Pablo Montanari y Lucia Montanari. Gracias a la Profesora Belén Calderón Roca de la Universidad de Málaga por sus consejos.

2 https://orcid.org/0000-0001-6716-7620. 
décadas sobre la capacitación de los profesores, y se reflexiona acerca de la profesionalidad del docente, su tenacidad, aspiraciones y nuevos horizontes.

Palabras clave: Currículo; Reformas educativas; Historia; Educación para la ciudadanía; Educación patrimonial; Formación docente.

Abstract: The article identifies the key stages that have characterized the education reforms in Italy, starting from the nineteenth century school programs up to the recent regulations on Civic Education and heritage education. In the last twenty years, various reforms (Moratti 2003, Gelmini 2008, Buona Scuola 2015) have caused significant changes to curricular programs still in place nowadays, like the abolition of the fifth year exam in primary school, the compulsory internships during secondary school, the compulsory and permanent teacher training programs aimed at teachers still in service; until the 2019 law on Civic Education as a separate subject and included in the programs of all levels, the introduction of which took place in the academic year 2020/2021. Taking into consideration the teaching practices conducted in recent decades, the conclusion of the essay will focus on teacher training and teacher professionalism: persistence, aspirations and new horizons.

Keywords: Curriculum; Education Reforms; History; Civic education; Heritage education; Teacher training.

SUMARIO: 1. Empezamos desde lejos. La Historia de los Programas escolares del siglo XIX. 1.1. Las reformas educativas después de la Unidad. 1.2. Nuevo siglo, nuevo comienzo, hasta los años Treinta. 1.3. La situación en la segunda mitad del 1900. 2. La evolución de los años noventa y el comienzo del nuevo siglo. 2.1. El punto de inflexión de las Indicaciones Nacionales. 3. Historia, ciudadanía y patrimonio. Entre anhelos, realizaciones efectivas y perspectivas. 3.1. Conocimiento del patrimonio y formación del profesorado, entre la investigación y la formación. 3.2. Las habilidades. Los temas y enfoques. 4. Algunas consideraciones finales. Persistencia y horizontes en la enseñanza de la Historia y la formación del profesorado. 5. Referencias bibliográficas.

\section{EMPEZAMOS DESDE LEJOS. LA HISTORIA DE LOS PROGRAMAS ESCOLARES DEL SIGLO XIX}

Para tener una perspectiva completa de los eventos que derivaron de las reformas, y del rol que asumió la disciplina histórica en relación a su enseñanza, en diferentes órdenes y grados, consideramos necesario ofrecer una visión general de disposiciones legales que se desarrollaron desde mediados del siglo XIX, hasta el contexto actual. Desde inicios del siglo XIX, cuando la Historia viene introducida como tema de estudio en los diferentes cursos educativos, esta es considerada una disciplina con significativa influencia sobre los jóvenes en formación.

En Italia, y en especial, después de la Unidad de 1861, la disciplina histórica se imparte con el propósito de crear una cultura nacional fuerte y arraigada. Se propuso el objetivo de hacer crecer y madurar los niños, futuros ciudadanos, partiendo del sentido de pertenencia y del patriotismo. 


\subsection{Las reformas educativas después de la Unidad}

En el momento de la Unificación, Italia era un país muy retrógrado, la crisis socioeconómica que reinaba en la casi totalidad del territorio no había dejado considerado casi la necesidad de instruirse. Aunque algunas regiones del norte, como Piamonte, Lombardía y Véneto, habían experimentado algunas innovaciones en el ámbito escolar, la mayor parte de la población era analfabeta y pocos conocían correctamente el idioma italiano. Es más, no sabían ni leerlo ni escribirlo (Panciera y Zannini, 2013, p. 72). La escuela era retrógrada y conservadora, e indebidamente proyectada hacia la democracia y la equidad, por lo que continuaba perpetuando las desigualdades.

En el 1847 se crea el Ministerio de Educación, pero solo después de la mitad del Ochocientos se empieza a tomar forma la idea de una Escuela Estatal más concreta (Aquiloni, 2016); una unificación del sistema que, como veremos, no fue sencilla. Los diferentes grados de educación, efectivamente, han sido reformados como si fueran entidades aisladas, olvidando la visión global de todo el complejo educativo.

Con la Ley Casati ${ }^{3}$ (1859) empezó una unificación del sistema de enseñanza Primaria en el Reino de Cerdeña, promulgada con la idea de una educación todavía elitista. No se concedía mucha importancia a los primeros años de formación escolar. Y la Guardería (hoy llamada Escuela Infantil) no estaba incluida en el plan de estudios, porque la consideraban sencillamente una alternativa para permitir trabajar a las familias con niños pequeños. La Escuela Primaria empezaba a partir de los seis años y se desarrollaba en dos ciclos bienales: uno inferior, obligatorio y gratuito, y uno superior, presente solo en los municipios con alta densidad (más de 4000 habitantes). Según los Programas Mamiani del 1860, la enseñanza se basaba principalmente en la técnica leo-escribo y la aritmética elemental; mientras que la Historia se empezaba a introducir solo en el grado superior y se refería en su mayor parte a la Historia Sagrada.

Con algunas referencias relativas a los deberes del ciudadano, así como a los hechos más sobresalientes de la Historia Nacional y algunos elementos de Geografía (cómo estaba dividido el globo terráqueo, los principales Estados y capitales de Europa y una corta descripción de Italia), estos argumentos eran solamente "leídos», ya que no requerían un estudio profundo, "de manera que se entienda que en las Escuelas Primarias no se quiere enseñar ni ética, ni derecho constitucional, ni la Historia de Italia, ni física, ni Geografía, pero solo dar elementales nociones que los jóvenes sean capaces de comprender y que sean útiles, bien quieran seguir con los estudios o bien que abandonen la escuela ${ }^{4}$.

${ }^{3}$ Ley 13 noviembre 1859, n. 3725 «Ley de organización de la escuela en el reino de Cerdeña».

4 «Programas para la Escuela Primaria» adjuntos al Reglamento del 15 de septiembre de 1860 [https://www.museodellascuola.it/wp-content/uploads/2020/04/01-i-programmidel-1860.pdf]. 
Amplio espacio, por el contrario, tendrá la enseñanza en la Escuela Secundaria y los estudios superiores. Se distinguían dos cursos: el clásico, que era caracterizado por una escuela Secundaria de cinco años y un Liceo de tres, y el Técnico, subdividido en tres años de escuela técnica y otros tres de Instituto Técnico. También en estos casos la Historia tenía su lugar solo en el ámbito clásico (humanístico), como elemento de soporte para el estudio del Griego y del Latín (Bruni, 2005, p. 22). Además de esta falta de óptica vertical, también la formación de los docentes en muchas ocasiones fue descuidada, como si esta no fuese un elemento determinante en la calidad del sistema educativo en su totalidad. Una tercera opción, además de los dos cursos que hemos mencionado, era la que llamaban Normal, dirigida a jóvenes con edades que oscilaban entre quince y dieciséis años, organizada con un plan de estudios de tres años que incluía disciplinas básicas (Italiano, Matemáticas, Ciencias, Historia y Geografía), y algunas rudimentarias nociones de enseñanza aplicada.

La Universidad era una opción casi exclusiva de los estudiantes del Liceo y se contaban muy pocas facultades entre las cuales mencionamos: Teología, Ley, Medicina, así como las recién creadas «Filosofía y Letras» y «Ciencias físicas, Matemáticas y Naturales".

Con la unificación de Italia en 1861, este sistema se extendió a todo el estado italiano, descuidando algunos aspectos fundamentales, como el hecho que este tipo de escenario había nacido en un específico ambiente sociocultural y no tenía en cuenta las exigencias tan diversas del territorio italiano en su totalidad. Además de la preparación de los docentes de Primaria, que muchas veces resultaba deficitaria, se acrecentaban las diferencias de un plan de estudios breve y sencillo para los alumnos de clase baja, y uno más completo para los hijos de la burguesía y de la nobleza. Se perpetuaban así los problemas de una sociedad rígidamente estratificada, predominantemente campesina y analfabeta, que una escuela de buena calidad habría contribuido a mejorar y ponerle remedio.

La Ley Coppino ${ }^{5}$ (1877) instituyó la obligatoriedad de escolarización hasta los nueve años de edad (es decir, los primeros tres de los cinco años de Escuela Primaria), estableciendo sanciones para los que no la respetasen. Las instituciones aumentaron, también se inició la implementación del mecenazgo escolar ${ }^{6}$, para favorecer la escolarización de los alumnos que pertenecían a las clases trabajadoras. No obstante, las difíciles condiciones socioeconómicas eran tan graves que no permitían la realización del programa. Además, se asignó a los municipios la carga del mantenimiento y el mejora de las escuelas, por lo que los efectos de la reforma fueron extremadamente lentos.

Las indicaciones ministeriales dadas por los Programas Gabelli (1877), integradas sucesivamente por Bacelli (1894), delineaban para las Escuelas Primarias

${ }^{5}$ Real Decreto 15 julio 1877, n. 3961 «Ley de educación Primaria obligatoria».

${ }^{6}$ Real Decreto 6 febrero 1888, n. 5292 «Reglamento único para la educación Primaria». 
unas directrices más ideológicas que de contenido, mientras que se volvieron más detalladas para los grados de escuela sucesivos. En lo que respecta a la Historia, la enseñanza elemental se basaba sobre todo en " "cuentos animados y aptos para hablar a la imaginación", anclados a aspectos de la vida cotidiana que son fáciles de memorizar (...) para entender como "nuestro país contribuyó a la civilización en el mundo" inspirando con su ejemplo el sentimiento del deber en los jóvenes, la devoción al bien público y el amor por la patria» (Panciera y Zannini, 2013, p. 73). Un plan de estudios más detallado tenían las escuelas de Secundaria, en las cuales «abrazando todo el evento histórico humano, terminaban constituyendo un catálogo del conocimiento histórico que representaba [...] la única reconstrucción posible del pasado» (Ibid.).

Se evidencia pues, la influencia del positivismo en el intento de laicizar la escuela estatal, agregando disciplinas como educación física y cívica, destacando así la función educadora de una escuela llamada a transmitir una educación también ideológica y política, en un país todavía profundamente dividido.

\subsection{Nuevo siglo, nuevo comienzo, hasta los años Treinta}

Durante la década de 1900 empezaron una serie de intervenciones ministeriales sobre los currículos que sustancialmente tenían como objetivo complacer las aspiraciones políticas de quienes en ese momento ostentaban el poder (y todavía hoy en día la situación no es estable, aunque en constante evolución). Así, los planes de estudios y libros de texto se transformaban en importantes instrumentos ideológicos que permitían dirigir la capacitación y la difusión de valores específicos.

Un período clave para el sistema escolar italiano fue el de la edad giolittiana (1903-1914). En las primeras dos décadas del siglo pasado, en efecto, en Italia se observó una democratización difusa y una renovación sustancial de las instituciones públicas. Con la Ley Nasi ${ }^{7}$ (1902) y la de Orlando $^{8}$ (1904) el Estado se hizo cargo de la protección del patrimonio cultural y de la instrucción en los niveles de escuela superior. Se extendió la obligación de asistir a la escuela hasta los doce años, instituyendo un plan de estudios popular de corte profesional que duraba dos años, después de los cuatro de Primaria, para aquellos que no hubiesen seguido estudiando en el Liceo o en la escuela técnica. Los programas Orestano (1905) resultaron ser más densos y detallados que los anteriores y se podría decir que establecían una distinción entre la enseñanza "formal», para los hijos de la burguesía y la "práctica» de

7 Ley 12 junio 1902, n. 185 «Disposiciones relativas a la protección y conservación de monumentos y objetos de valor artístico o antiguo». La primera ley real sobre los Bienes. Estableció un "Catálogo Único» nacional (obras artísticas e históricas del estado) e introdujo el derecho de tanteo por parte del estado junto con la prohibición de la exportación de bienes.

${ }^{8}$ Ley 8 julio 1904, n. 407 «Disposiciones de Orlando». 
las clases trabajadoras, que seguirían después una dirección hacia la escuela complementaria y, sucesivamente al trabajo, perpetuando nuevamente las diferencias mencionadas anteriormente.

Con la Ley Daneo-Credaro ${ }^{9}$ (1911) se trató de mejorar la cantidad y calidad de las escuelas, revisando los programas y los planes de estudios y, sobre todo, se responsabilizó al Estado de los problemas relativos a la educación, inclusive los de la Escuela Primaria. Se establecieron Consejos Provinciales para fomentar el patrocinio escolar y dar mayores posibilidades a las clases sociales más desfavorecidas (Barbieri en Sorzio, 2011, p. 76).

En cuanto a los docentes, generalmente mal pagados, sin preparación y sin autoridad ante las administraciones municipales, se establecieron fondos para ayudar a los municipios más pobres y mejorar las condiciones de los docentes en las zonas rurales, aumentándose los salarios (y equiparando incluso los de hombres y mujeres); se establecieron cursos para el desarrollo de las carreras profesionales de los docentes. En la formación, las escuelas normales fueron fuertemente criticadas, no solo por las muchas lagunas en el plan de estudios, sino sobre todo por su demostrada evidente ineficacia. Los estudiantes que eligieron la escuela normal eran a menudo los peores del grado escolar anterior, provenían de un entorno cultural pobre y no estaban muy motivados. Hacia 1910, la Asociación Nacional de Estudios Pedagógicos reconoció la necesidad de ampliar y fortalecer el plan de estudios y las prácticas docentes, no solo en términos de tiempo, sino también en términos de contenido y actividades de capacitación. Ya no podían limitarse a la presentación de alguna teoría o a la observación de la actividad de otros profesores, sino que se consideró esencial interactuar con ellos y con la clase, de forma activa y proactiva (Zago, 2005, pp. 153-154).

El estallido de la Primera Guerra Mundial hizo pasar en segundo plano el problema del desarrollo escolar italiano, lo que provocó graves desaceleraciones. Con Benedetto Croce $^{10}$ (1921) finalizó el último intento de reforma orgánica de los programas, aunque las consecuencias no fueran tan evidentes, el esfuerzo activado fue el de «asignar a la Historia la tarea de capacitar al aprendiz para recopilar y conocer los hechos y conectarlos en una interpretación global» (Panciera y Zannini, 2013, p. 74), y así liberar a la disciplina de la idea de una Historia objetiva, la "oficial», con la esperanza de alejarla de esa ideología demasiado arraigada. La incapacidad para superar este problema se hizo aún más evidente en los años siguientes.

${ }^{9}$ Ley 4 junio 1911, n. 487 «Disposiciones para la educación elemental y popular». Durante el ministerio de Giolitti, esta ley nacionalizó la Escuela Primaria, que anteriormente había sido administrada por los municipios.

${ }^{10}$ Croce fue ministro de Educación en el quinto y último gobierno de Giolitti (1920-1921). 
En la década de 1920, con el auge del fascismo, la Reforma Gentile ${ }^{11}$ (1923) consideró por primera vez, desde una perspectiva vertical de un sistema único e integrado, la idea de una Escuela Infantil (entonces llamada "grado preparatorio») un curso de tres años basado en actividades lúdicas y recreativas, sin embargo, dirigido hacia una primera adquisición de las reglas de conducta y disciplinas académicas básicas. La Escuela Primaria permaneció dividida en grado inferior y superior, seguida de la Escuela Intermedia Inferior. Por tanto, la obligatoriedad de escolarización se amplió hasta los catorce años y permitió a los jóvenes adquirir las competencias suficientes para continuar sus estudios (en las Escuelas Secundarias: Escuela Secundaria Clásica, Escuela Secundaria Científica, Instituto Técnico o de maestría).

En los años anteriores, el pedagogo Radice ${ }^{12}$ desarrolló un modelo centrado en la idea de una escuela activa y pacífica, proponiendo una enseñanza basada en la narración y vinculada a personajes simbólicos, que aún tomaba en consideración la necesidad de dar respuestas educativas a las necesidades de los niños. Este sistema pronto se vio asfixiado por la necesidad ideológica del fascismo de adoctrinar y militarizar a la juventud italiana. En 1929 se introdujo un único libro ${ }^{13}$ de texto y se elaboraron nuevos programas con el Ministro Ercole ${ }^{14}$ (1934) que, en lo que a Historia se refiere, se centraron en la exaltación de la antigua Roma, en la sobreestimación de las empresas coloniales italianas, en algunas figuras relevantes del Renacimiento y sobre episodios gloriosos de acción colectiva e individual para revitalizar el nacionalismo. Esta elección se debió a la necesidad de promover el fascismo y que marcó una clara separación de las iniciativas anteriores. En 1939 se emitió la llamada "Carta Escolar» ${ }^{15}$ con la que se intentó un alejamiento oficial de ese enfoque gentiliniano considerado demasiado selectivo (Barbieri en Sorzio, 2011, pp. 77-79). El estallido de la Segunda Guerra Mundial marcó un nuevo revés.

${ }^{11}$ Una serie de disposiciones (Reales Decretos) emitidas entre 1922 y 1924 que reformaron todo el sistema escolar (de todos los ordenes y niveles) bajo la evidente influencia del ascenso fascista.

12 Para más información ver Martorana, Ambrosi y Savarino (2001, noviembre). http:// www.dubladidattica.it/lomradice.html

13 Ley 7 enero 1929, n. 4, "Didáctica del texto único». La ley de introducción del libro de texto fue aprobada a finales de los años treinta y entró en vigor con el curso escolar 1930-31. Para más información, ver "La enseñanza del texto único» (2001, enero y febrero). La Nuova Resistenza Unita. https://www.casadellaresistenza.it/pubblicazioni/nuova_resistenza_unita

14 «Nuevos programas» de 1934 (Radice). https://www.museodellascuola.it/wp-content/ uploads/2018/03/07I-programmi-del-1934_PREMESSA.pdf

${ }^{15}$ Ley 1 julio 1939, n. 899 «Protección de cosas artísticas o históricas» (Bottai). http://www. dirdidatticamelia.it/htm/storiascuo/1920-1940/Web/carta\%20scuola\%20.htm 


\subsection{La situación en la segunda mitad del 1900}

En Italia, liberada del nazi-fascismo, el control centralizado y estatal de la educación pública aún perpetuaba ese modelo basado en la filosofía gentiliniana. El punto de inflexión llegó en 1945, cuando la comisión aliada para la educación pública, bajo el liderazgo del pedagogo estadounidense Washburne ${ }^{16}$, instituyó los nuevos currículos de Guardería y Escuela Primaria. Inspirado en la ideología de Dewey y Kilpatrick ${ }^{17}$, este nuevo enfoque marcó una clara desviación de la rigidez y la intolerancia que había invadido el sistema escolar en años anteriores. En el corazón de esta nueva organización, se incluía el desarrollo de conocimientos básicos de Italiano y Matemáticas, que se abordarían de manera individualizada tanto como fuera posible. En cuanto a la Historia, considerada inseparable de la Geografía, tuvo un papel importante en todo el recorrido escolar, a partir de los primeros años de la Escuela Primaria. Se anuló cualquier manifestación de nacionalismo para favorecer un estudio más profundo de los pueblos del pasado, revividos a través del enfrentamiento con el contexto actual y como base fundamental para redescubrir esos ideales de hermandad y respeto que mucho se habían alejado con las filosofías fascistas. Las actividades también deberían haber incluido momentos creativos, centrados en los intereses y preferencias de los alumnos. Precisamente por su flexibilidad y alejamiento de los cimientos bien establecidos del sistema anterior, estos programas no tuvieron la efectividad deseada (Panciera, 2016, p. 39).

En 1955, el ministro demócrata cristiano Ermini reformó nuevamente el plan de estudios aprobando los nuevos currículos de la Escuela Primaria ${ }^{18}$ con un enfoque marcadamente católico. También se redactaron notas operativas para la Escuela Infantil basadas en un supuesto sentido común educativo (similar al de una madre) y no en prescripciones metodológicas (Bonetta, 1977, pp. 188-189). La Historia perdió su prestigio a favor de la educación moral y cívica, que en ese período se sintió como una necesidad, dada la dinámica del régimen anterior. Junto a la Ciencia y la Geografía, la Historia se redujo a un elemento de conocimiento genérico del mundo para esa «transición paulatina de la intuición global del entorno a los primeros análisis de los contenidos culturales detectados en el propio entorno» (Panciera, 2016, p. 40). El conocimiento histórico tuvo que limitarse a resaltar los grandes

${ }^{16}$ En la filosofía de Washburne, una buena planificación escolar debería haber tenido una parte común, centrada en el desarrollo de conocimientos técnicos básicos, y una parte libre y creativa, para fomentar la experiencia de nuevas habilidades basadas en las aptitudes específicas del individuo. Véase Washburne, 1940. Para más información ver Gelmi, 2019.

17 Dos educadores estadounidenses del siglo xx. Colegas del Teachers College de la Universidad de Columbia desde 1909, se dedicaron al estudio de la dinámica de la educación, en particular el papel de la libertad de experiencia y descubrimiento en la formación del niño.

18 D. P. R. 14 junio 1955, n. 503, «Programas educativos e instrucciones relacionadas para Escuelas Primarias, públicas y privadas». 
acontecimientos del pasado para reforzar el patriotismo y el resurgimiento nacional, perdiendo toda referencia al presente. Esto llevó a los profesores a seguir las páginas de los libros de texto reduciendo la competencia en el campo histórico a la mera narración y memorización de una lista de hechos, no muy lejos de lo ocurrido anteriormente. La ralentización de Italia, con respecto al avance de la disciplina en el resto de Europa se hacía cada vez más evidente.

En la década de 1960 se introdujeron algunas reformas ${ }^{19}$ que intentaron socavar la idea de una escuela selectiva basada en el nivel socioeconómico de las familias de los alumnos. Esta apertura, sin embargo, supuso un aumento notable del número de alumnos que, antes, se habrían encauzado hacia las escuelas profesionales o incluso hacia el trabajo manual, ahora podrían haberse matriculado en una escuela media, cuyo plan de estudios se basaba en disciplinas mucho más teóricas, sin haber proporcionado a los profesores la preparación adecuada para gestionar tal cambio. La falta de una reforma integral del plan de estudios terminó por poner de relieve las diferencias sociales.

La situación de los profesores ciertamente no era la más favorable: solos, frente a clases masificadas (hasta 40-45 alumnos), a menudo obligados a utilizar métodos tradicionales y sin el apoyo de las familias (Barbieri en Zago, 2008). De hecho, la formación de los profesores de la Escuela Primaria seguía siendo competencia del Instituto de Enseñanza, mientras que para los de la escuela Secundaria el reclutamiento se basaba en concursos que evaluaban solo los conocimientos disciplinares (obtenidos con un título universitario en el campo específico), sin atender a actitudes psicopedagógicas, ni a destrezas profesionales. Maestros que desde el punto de vista cognitivo estaban bien preparados, pero carentes de habilidades didácticas que, con suerte, se habrían adquirido con la experiencia. El único curso de nivel universitario para la formación del personal escolar fue el Diploma de Supervisión Escolar, impartido en la Facultad de Educación. Una trayectoria académica de cuatro años dedicada a los estudiantes que habían completado el instituto de maestría de cuatro años y que no pudieron inscribirse en un curso académico regular, por no haber obtenido un diploma de cinco años. A finales del siglo XX, con la creación de la carrera de Educación Primaria y de la Escuela Interuniversitaria de Especialización para la Formación del Profesorado de Secundaria (SSIS), se concretó una idea de formación, también a nivel universitario, para futuros profesores (Barberi en Sorzio, 2011, pp. 73-101).

Los nuevos programas de la Escuela Secundaria ${ }^{20}$ (1979) no difirieron mucho de los de diecisiete años antes; estaban enfocados a los contenidos disciplinares,

19 Ley 31 diciembre 1962, n. 1859, «Establecimiento y organización de la escuela Secundaria estatal».

20 D. M. 9 febrero 1979, «Programas, horarios y exámenes para la Escuela Secundaria estatal». 
no dejaban vislumbrar un nuevo marco psicopedagógico y ni siquiera un enfoque metodológico actualizado (Panciera, 2016, p. 40). La Escuela Primaria, en cambio, sufrió afortunadamente un destino diferente: los Nuevos programas de $1985^{21}$, inspirados en el modelo anglosajón basado en la psicología cognitiva y la teoría curricular, destacaron un gran esfuerzo positivo en la delimitación, en primer lugar, de los fundamentos pedagógicos e, inmediatamente después, de los contenidos disciplinares ( $/$ bid.). Programas amplios que deberían haber permitido a los profesores planificar el plan de estudios de acuerdo con las necesidades de formación de los alumnos, las expectativas sociales y los objetivos educativos. En particular, en lo que respecta a la disciplina histórica, bajo la influencia de nuevos estímulos derivados de la investigación, la historiografía y la docencia, el valor y los objetivos educativos se redefinieron en la perspectiva de esa "Nueva Historia», ahora difundida por toda Europa, que destacó el papel mediador entre pasado, presente y futuro como capaces de "estimular y desarrollar en los niños la transición de la cultura vivida, absorbida directamente en el medio vital, a la cultura como reconstrucción intelectual $»^{22}$. El punto de partida del proceso formativo pasó así a ser la experiencia del niño que debía ser reelaborada mediante la acción de una enseñanza activa y participativa, que ya no podía limitarse a la mera transmisión-memorización de contenidos. El foco ya no era la asimilación de conocimientos, sino la adquisición de herramientas conceptuales y procedimentales fundamentales para el crecimiento global de los futuros ciudadanos (Panciera, 2016, p. 41).

A principios de los Noventa también se estableció la Escuela Infantil estatal, seguida de las respectivas directrices ${ }^{23}$, que conllevaron importantes actualizaciones, más acordes con las que se habían estabilizado en otros países europeos. Ya no se trataba de un "preescolar», sino de un sistema con su propia planificación y autonomía pedagógica, también impulsado por experiencias como las de las escuelas extranjeras (a menudo llamadas Kindergarten). La superación de la idea de la Guardería como sustituto temporal de las funciones maternas se vio reforzada aún más por el cambio en el nombre de la propia escuela, ya no "Guardería» sino «Infancia».

Sin embargo, todo este nuevo aparato no tuvo en cuenta la preparación de los profesores, decididamente inadecuada para cambios tan radicales. Es evidente que las iniciativas en el ámbito organizativo no han sido seguidas por el mismo espíritu de innovación en materia de formación del profesorado. El nuevo perfil profesional del docente requería no solo habilidades cognitivas, sino sobre todo metodológicas,

${ }^{21}$ D. P. R. 12 febrero 1985, n. 104, «Aprobación de los nuevos programas didácticos para la Escuela Primaria».

22 «Programas para la Escuela Primaria» (D. P. R. 104/85).

${ }^{23}$ D. M. 3 junio 1991, «Directrices para la actividad educativa en las escuelas infantiles estatales». 
a las que difícilmente se podía acceder sin una buena trayectoria de estudios que incluyera también un curso universitario.

Los programas de 1985 no tuvieron la aplicación deseada, pero el contenido de estos, deliberadamente genérico para favorecer la iniciativa de los docentes, sirvió de inspiración para lo que luego se convertirán en las Directrices Nacionales. Para la disciplina histórica, por ejemplo, se sugirió fijar los hechos más relevantes del pasado y profundizarlos junto a los personajes decisivos para la Historia italiana. Sin embargo, esto no tuvo el resultado deseado, que tenía que ser una renovación sustancial en la docencia, sino que terminó volviendo al tratamiento simple y sin problemas de los contenidos de la filial. Esta contradicción puso de relieve la falta de preparación de los docentes con respecto al perfil profesional esperado. No existía un sistema de actualización generalizado, sino iniciativas aisladas y mal distribuidas en el territorio. Algunos docentes intentaron el camino de la renovación, pero estos fueron casos excepcionales que tuvieron poco impacto en la difusión de buenas prácticas.

\section{LA EVOLUCIÓN DE LOS AÑOS NOVENTA Y EL COMIENZO DEL NUEVO SIGLO}

Reconocida la necesidad de una reforma global del sistema, que hasta ese momento había visto solo cambios parciales que muchas veces no habían tenido en cuenta las repercusiones en los grados escolares anteriores o posteriores. En las postrimerías de los noventa, con el Ministro Berlinguer ${ }^{24}$ (1997), se empezó a pensar en una configuración pedagógica progresiva y coherente. Numerosas iniciativas atestiguan el impulso revolucionario de esta nueva reforma que suscitó, no obstante, no pocas controversias. En concreto, como hemos visto en el devenir de nuestro discurso, la Historia, propia de sus hallazgos en el campo ideológico y político, fue una de las disciplinas sobre las que más se discutió. Por una parte, estaban los que querían dar mayor importancia a los hechos más recientes, precisamente para facilitar la reflexión histórico-política sobre las condiciones actuales. Y, por otro lado, estuvieron quienes lo consideraron "peligroso», dada la explotación que muchas veces se hizo de ese período, además de la imposibilidad de reconciliación con la estructuración cronológica, anterior (Cajani, 2019, pp. 5-6). Con la Ley cuadro del $2000^{25}$, se inició la subdivisión de los ciclos educativos en solo dos grandes bloques: una Primaria, de seis años, y una Secundaria, de otros seis años de los cuales los tres primeros fueron iguales para todos, mientras que los últimos tres años eran

${ }^{24}$ Ministro de Educación del gobierno Prodi, emitió una serie de leyes entre 1997 y 2000 para reformar la escuela italiana.

${ }^{25}$ Ley 10 febrero 2000, n. 30 «Ley quadro para la reorganización de los ciclos de educación superior». 
especializados en función de la dirección elegida. En el año 2000 el trabajo continuó con el nuevo Ministro Tullio De Mauro ${ }^{26}$, quien pasó a delinear los nuevos planes de estudio y el contenido de los diferentes ciclos, con los grupos de trabajo específicos para cada disciplina. En particular, la Historia se fusionó con la Geografía y las ciencias sociales, que siempre se consideraron interconectadas, pero que luego adquirieron una mayor autonomía a lo largo de los años de estudio. Para la Historia, bajo la influencia de nuevas investigaciones internacionales, se decidió abordar el pasado del hombre en un sentido cronológico, intentando quitar esa mirada típicamente eurocéntrica (por no decir italocéntrica) en favor de un enfoque multinacional (Cajani, 2019, p. 10). Un cambio tan importante, que en opinión de muchos habría desviado la atención de esa dimensión de identidad nacional. A pesar de ello, De Mauro todavía intentó que se aprobara el nuevo plan de estudios, pero el gobierno cayó antes de su implementación.

Como se anticipó, a partir de finales de los noventa, la alternancia de fuerzas políticas en el gobierno provocó una sucesión de demoliciones de los programas propuestos por el antecesor, para reformular otros nuevos, en busca de resultados llamativos en el corto plazo (Fiore, 2001). Esta contradicción terminó ralentizando, si no empeorando, el desarrollo del sistema escolar, que requería de escalas de tiempo más largas para poder ver resultados significativos.

Con la Reforma de Moratti27 (2003) el gobierno de Berlusconi intentó, de hecho, revocar lo que se había hecho al intentar nuevamente la empresa de una renovación global. Bajo el control del gobierno de centro derecha, el perfil del alumnado se perfilaba seleccionando e identificando las competencias que el alumno debería haber tenido al finalizar la escolaridad obligatoria. Las Escuelas Primarias y las de segundo grado (antiguas escuelas intermedias) se fusionaron en Institutos Integrales bajo el control de un solo administrador escolar, siendo así concebidos como un solo bloque curricular (Cerini, 2002). La Historia, en particular, adquirió su autonomía, separándose de la Geografía y de las ciencias sociales. Con las Indicaciones Nacionales ${ }^{28}$ (2004) se vislumbraba un camino vertical que ya a partir de la tercera Primaria, con la aparición del hombre, continuaba en un continuum progresivo, atravesando las grandes civilizaciones antiguas hasta la caída del Imperio romano,

${ }^{26}$ Ministro de Educación del Gobierno Amato (2000-2001) bajo el cual se publicaron los nuevos, y extremadamente detallados, planes de estudios escolares básicos, pero aquí también el gobierno cambió muy rápidamente.

27 La Ley 53/2003, como hemos visto, se refiere a la reforma de la escuela con la que se elaboraron diversos borradores de documentos, entre los que se encuentran: Recomendaciones e Indicaciones Nacionales para la Escuela Primaria y Secundaria de primer grado, el perfil educativo, cultural y profesional del alumno al finalizar el Primer Ciclo de Educación (6-14 años).

${ }^{28}$ Con la Ley n. 53/2003, pero sobre todo con el D. Lgs. n. 59/2004, se introducen las "Indicaciones Nacionales para Planes Personalizados» (y Actividades Educativas en Jardín de Infantes, Escuela Primaria y Secundaria primer grado adjunto). 
para luego llegar al final del siglo XIX, al finalizar la escuela Secundaria inferior. Contenidos que, aunque con algunas excepciones, evocaban ese sentimiento eurocéntrico y nacionalista del que tanto quería alejarse De Mauro.

En 2005 se emitieron las Normas Generales para el segundo ciclo ${ }^{29}$ en el que se identificaron «objetivos específicos de aprendizaje», objetivos que, una vez más, perseguían «la formación intelectual, espiritual y moral, también inspirados en los principios de la Constitución, el desarrollo de la conciencia histórica y pertenencia a la comunidad local, a la comunidad nacional y a la civilización europea» ${ }^{30}$. En lo que respecta a la Historia, por tanto, se retomó todo el currículum del primer ciclo, profundizando algunos pasajes cuyas referencias eurocéntricas eran evidentes.

\subsection{El punto de inflexión de las Indicaciones Nacionales}

Por su transitoriedad y su evidencia ideológica, las primeras Indicaciones $\mathrm{Na}$ cionales marcaron un retorno al paradigma decimonónico, donde se concedía gran importancia al descubrimiento de los orígenes de los pueblos y las consiguientes diferencias entre las sociedades actuales. Una Historia, cuya función estaba evidentemente ligada a la construcción de la identidad, pero que, en realidad, se reducía a un camino secuencial y trasmisor. Muchos criticaron este nuevo enfoque, visto como una involución con respecto a los avances de los programas de 1985 y aún hoy sin solucionarse (Panciera, 2016, p. 44).

Con el ascenso del gobierno de centro izquierda de Prodi, el ministro Fioroni actualizó las Indicaciones ${ }^{31}$ (2007) bloqueando lo hecho para el segundo ciclo de educación por la comisión anterior. Menos estructurados que en los planes de estudio anteriores, las nuevas indicaciones presentaban los contenidos de forma más genérica y no articulada año por año. El programa de Historia se vinculó con el de Geografía, conformando el «conocimiento único» más amplio del área históricogeográfica, que también incluyó aspectos de aquellas ciencias sociales para las cuales no se previeron objetivos específicos. Se habían sumado muchos detalles a la Historia del siglo XX, potenciando siempre aspectos vinculados al desarrollo de la identidad nacional, a pesar de que las premisas indicaban la necesidad de favorecer una dimensión global y la reflexión sobre una realidad cada vez más heterogénea y multicultural, en la que a estas alturas también estaba Italia (Cajani, 2019, pp. 2528).

29 D. Lgs. 17 octubre 2005, n. 226, «Normas generales y niveles esenciales de desempeño del segundo ciclo del sistema educativo y formativo» de conformidad con el artículo 2 de la Ley $53 / 2003$.

30 Ibidem, artículo 1, punto 3.

31 D. M. 31 julio 2007, "Directrices nacionales para el plan de estudios de las escuelas preescolares y de primer ciclo». 
Lamentablemente, incluso en este caso, los resultados no fueron los esperados, de hecho, en la mayoría de las escuelas, solo se siguió "haciendo» Historia antigua sobre la base de esquemas repetidos. "Hacer Historia», de hecho, es un término que muchas veces se ha asociado con la "realización de actividades relacionadas con la historiografía, sin distinguir en cuál de sus sectores disciplinarios fundamentales [investigación, docencia y difusión]. Un entorno creativo amplio y polifacético, pero desprovisto de límites, en el que los aspectos investigativos, educativos y comunicativos deben vincularse e integrarse recíprocamente» (Dondarini, 1999, p. 153), pero en muchas ocasiones no se corresponde con la situación real. Las distintas localizaciones institucionales, respectivamente el ámbito académico, escolar y de los medios de comunicación, no parecen integrarse en una red unitaria, coherente y rentable de relaciones y comunicaciones, y ello se evidencia en las distintas velocidades y direcciones con las que estas tres áreas se han desarrollado y hasta el día de hoy siguen evolucionando.

Con la Reforma Gelmini32 se dio un «respiro» a las indicaciones del currículo del Primer Ciclo para retomar el trabajo interrumpido cuatro años antes en el Segundo Ciclo, distinguiendo dos trayectorias en bachillerato: Técnico y Profesional. La fusión de la Historia con la Geografía se hizo oficial con la presencia de un solo grado en los dos años de la escuela Secundaria, y al eliminar por completo la Geografía de los otros dos sistemas escolares. Algunos núcleos temáticos se redujeron (especialmente los del pasado más remoto), mientras que otros presentaron mucho más contenido que antes, incluso con elementos extraeuropeos. No obstante, aún lejos de una visión global de la Historia, los currículos de Historia fueron concibiéndose un poco diferentes según el tipo de escuela para ser más «funcionales» y coherentes con el camino tomado, aunque la implementación dejaba mucho que desear (Cajani, 2019, pp. 29-30).

Unos años más tarde, Profumo intentó completar el trabajo concentrándose en los dos últimos años de la escuela Secundaria y promulgó las Directrices específicas $^{33}$ (2012). No muestran listados de conocimientos específicos, sino indicaciones generales que también dejan espacio para análisis y reflexiones en profundidad de carácter demográfico, social y cultural. Unos meses después, la publicación de las Nuevas Indicaciones Nacionales ${ }^{34}$ también pretendía renovar el Currículo del Jardín de Infancia y Primer Ciclo Escolar, y al que todavía nos referimos hoy para el diseño

32 Serie de leyes promulgadas después la Ley 6 agosto 2008, n. 133, durante el gobierno Berlusconi (2008-2010), por el Ministro de Educación M. S. Gelmini.

33 Directiva 16 enero 2012, "Adopción de los Lineamientos para la transición al nuevo sistema de institutos técnicos» de conformidad con el artículo 8, párrafo 3, del D.P.R 15 marzo 2010, n. 88.

${ }^{34}$ D.Lgs 16 noviembre 2012, n. 254, «Reglamento que contiene directrices nacionales para el plan de estudios de la Escuela Infantil y el primer ciclo de educación». 
de cursos educativos en la escuela básica. No hay trastornos con respecto a lo esperado, pero en ellas se perfilan no solo los principales núcleos conceptuales de cada área temática (o campo de experiencia para el Jardín de Infancia), sino también los propósitos generales del sistema escolar, retomando a las directrices europeas e identificando el perfil del alumnado que debe conformar una escuela democrática, inclusiva y de calidad, teniendo en cuenta un contexto hoy profundamente transformado, heterogéneo y sumamente dinámico. El objetivo es poder dejar más espacio a la autonomía del docente, para poder también favorecer caminos transversales que favorezcan la inclusión de temas de actualidad con continuas referencias al pasado, presente y futuro. Una idea de enseñanza basada en el problem solving y el desarrollo de habilidades críticas que serían útiles para la adquisición de conocimientos clave para la vida (Panciera, 2016). Una visión en línea con las tendencias y actualizaciones consolidadas desde hace tiempo en el resto de Europa ${ }^{35}$. Así pues, la Historia se convirtió en uno de los ocho pilares del sistema de formación y tenía que apuntar a los aspectos fundamentales del pasado, razonando según las civilizaciones, pero también activando las habilidades de investigación histórica en la vida cotidiana, como la observación directa y la investigación de "los rastros encontrados en el Patrimonio documental, museístico, monumental y paisajístico» (Panciera, 2016).

En un país que ha encontrado recientemente su propia unidad territorial, es importante promover la unidad de la identidad nacional, pero también es cierto que Italia ya no es lo que era hace 160 años. Hoy en día son muchas las realidades sociales, económicas, culturales e ideológicas que la escuela debe acoger. A pesar de la cantidad de reformas que se han realizado a lo largo del tiempo y su gran variabilidad, el sistema escolar ha cambiado muy poco, especialmente en lo que se refiere a los contenidos (Cajani, 2019, pp. 38-39), que siguieron siendo sustancialmente eurocéntricos y, por lo tanto, partidarios del desarrollo de una perspectiva consecuentemente distorsionada de una Historia que, a menudo, tiene mucho más que contar, vista desde un punto de vista diferente.

\section{HISTORIA, CIUDADANÍA Y PATRIMONIO. ENTRE ANHELOS, REALIZACIONES EFECTIVAS Y PERSPECTIVAS}

El interés y la sensibilidad sobre temas referentes a la salvaguardia y tutela de los bienes ambientales y culturales que conforman el patrimonio, dependen sobre todo del nivel y de la calidad del conocimiento histórico de la sociedad. La atención

${ }^{35}$ Resumen en la más moderna Recomendación del PARLAMENTO EUROPEO y del CONSEJO de 18 de diciembre de 2006 sobre las competencias clave para el aprendizaje permanente (2006/962/CE). 
por las herencias del pasado se impone ya sea en el aspecto de la formación, como en la exigencia de percibirlas y valorarlas como recurso.

No es retórico asegurar que cada territorio se convierte en fuente de su Historia y, por lo tanto, también de los eventos de las comunidades que en él han vivido. No respetar sus características significa, no solo comprometer los equilibrios socioculturales, sino también perder las huellas de tales eventos identitarios.

Para un país, la salvaguarda, la recuperación y el valor de los bienes culturales incluyendo los bienes naturales, ambientales y paisajísticos, además de los históricos y artísticos, resulta un deber esencial para preservar su identidad y apreciar sus memorias como elementos constituyentes de una riqueza cultural compartida. Bajo esta luz, las escuelas pueden concurrir por y hacia una sensibilización necesaria, promoviendo, en colaboración con museos, archivos culturales y bibliotecas, el camino hacia un amplio conocimiento de los bienes presentes en su territorio. Los años ochenta italianos se caracterizaron por importantes cambios a nivel escolar bajo el aspecto de la educación patrimonial. En ese sentido, el mundo escolar es protagonista de innovaciones significativas con respecto al aprendizaje del ambiente y de los bienes culturales, como lo atestiguan los programas de educación Primaria de 1985, y la reforma de la clasificación del primer grado de instrucción de 1990. Un particular énfasis sobre la importancia de una alfabetización cultural a través (y no solo) de la educación de la imagen ${ }^{36}$. Tal y como puede leerse entre los objetivos de los programas antes mencionados, la educación de la imagen tiene como finalidad un proceso de madurez hacia el gusto estético, a través de una comprensión siempre mayor del arte y sus obras ${ }^{37}$. Por lo tanto, hay que disponer a los alumnos en condición de conocer la variedad de los bienes culturales de su territorio, ya que solamente de esa manera se les puede garantizar una efectiva educación patrimonial y un óptimo aprendizaje de sus expresiones. Igualmente importantes son los contactos con expertos de las secciones didácticas de los museos, así como la colaboración con Asociaciones e Instituciones culturales, como Italia Nostra y la IBC de la región Emilia-Romagna, como ejemplo (Branchesi, 2004).

En primer lugar, con respecto a los años setenta, la educación patrimonial llega a involucrar el primer nivel educativo. En este sentido, los programas de 1985 no solo introducen el nombre de educación de la imagen, sino que también atribuyen a esta última un papel importante en la educación del patrimonio. De hecho, la maduración del gusto estético hacia la obra de arte va de la mano del «disfrute» de un gran número de bienes culturales. Desde esta óptica, por lo tanto, es necesario crear oportunidades para que los estudiantes conozcan su territorio (y no solo) desde un punto de vista cultural. La arquitectura, los monumentos, las obras de

36 Introducido en lugar de «dibujar y escribir» de los programas de 1955.

37 D. P. R. 12 febrero 1985, n. 104 «Nuevos programas educativos para la Escuela Primaria». 
arte, los paisajes urbanos y naturales, los bienes tangibles e intangibles..., pueden representar elementos válidos a este respecto.

En segundo lugar, estamos asistiendo a una implementación de las secciones educativas dentro de los museos y las instituciones culturales. En este sentido, cada vez son más los lugares que se comprometen a promover talleres y proyectos para estudiantes y adultos ${ }^{38}$. Por lo tanto, podemos afirmar cómo el museo, de una institución cultural "limitada» a un lugar cerrado y definido, comienza a expandirse cada vez más en el entorno circundante, tanto en términos de diseño como en el propio sentido físico.

Entrando en la última década del siglo XX, podemos observar cómo diferentes experiencias, tales como las clases de patrimonio europeo, se están convirtiendo cada vez más en una realidad consolidada y generalizada. Posteriormente, en el 2006, el Parlamento y el Consejo Europeo introdujeron las competencias clave de la ciudadanía que deberían ejercer todos los Estados miembros. Al año siguiente, con las Indicaciones Nacionales para el currículum, el Ministerio de Educación inserta las ocho competencias clave entre los objetivos a perseguir al finalizar el primer ciclo educativo. Por lo tanto, podemos observar cómo, apenas dos años después del Año Europeo de la Ciudadanía ${ }^{39}$, las políticas nacionales de educación ya están actuando en términos de educación para la ciudadanía democrática (Ávila, Borghi y Mattozzi, 2009). Cabe destacar que, de entre las ocho competencias clave, el patrimonio cultural se encuentra entre las protagonistas. También en el mismo año se estableció que el 2018 debería ser el Año Europeo del Patrimonio para dar un mayor impulso al patrimonio cultural europeo y a las tendencias que han surgido en los últimos años. Se quiere fortalecer el sentido de identidad y pertenencia europea asignando un papel de liderazgo a la institución educativa.

Actualmente, el Ministerio de Educación, Universidad e Investigación (Miur) aporta una importante contribución al patrimonio cultural. Nos referimos al Programa Operativo Nacional (PON) del Ministerio -financiado por los Fondos Estructurales Europeos- que contiene las prioridades estratégicas en educación que se pueden alcanzar del 2014 al 2017. En particular, es el PON de 2017 referente al patrimonio cultural, artístico y paisajístico el que nos interesa. Este último, de hecho, evidencia la importancia de "concienciar a las alumnas y alumnos de su propio patrimonio [...] con el objetivo formativo de educarlos para protegerlo tutelarlo [...] potenciando el valor de su dimensión de bien común y el potencial que puede generar para el desarrollo democrático del país ${ }^{40}$. El Ministerio de Educación, a partir de una enorme financiación europea, invita a las escuelas a participar en esta

\footnotetext{
38 Véase Castello di Rivoli, Pecci Center en Prato y MART en Rovereto.

39 En 2005.

${ }^{40}$ Enlace PON, https://www.istruzione.it/pon/avviso_patrimonio-artistico.html
} 
iniciativa con el fin de educar a una ciudadanía activa, responsable y consciente, de su territorio.

En general, el programa registró una adhesión del 45,5 \% correspondiente a una suma entre las escuelas que presentaron el proyecto como particular o líder, y las que participaron en la red (online) ${ }^{41}$. Por lo tanto, este dato nos hace reflexionar sobre la abundante participación, aunque aún se necesiten emplear más incentivos. En este sentido, la formación -inicial y permanente-de los docentes es aún más imprescindible actualmente.

Por todos estos motivos, desde el Jardín de Infancia, el "patrimonio» resulta ser un elemento necesario y un vector de diálogo de gran valor educativo, capaz de abrir y proyectar el potencial de campos disciplinarios específicos, en horizontes cada vez más amplios y de aprovechar las herramientas de comunicación más actuales. Se convierte, tal y como sugieren las propias Indicaciones Nacionales, en una oportunidad para la adquisición y producción de conocimientos que estimulan la conquista de competencias y la construcción de conocimientos a través de experiencias específicas de investigación y docencia. Ello requiere la confluencia de dependencias y la convergencia de caminos, en un entrelazamiento interdisciplinario enriquecido también por actividades simbióticas entre la escuela y demás instituciones exteriores. Implica un uso sistemático de todas las herramientas de comunicación y, en particular, de las tecnologías telemáticas y de los soportes multimedia que se pueden usar en todo proyecto didáctico y de difusión. Desde la percepción y definición de los componentes y ubicaciones del "patrimonio», hasta la adquisición específica relativa a sus componentes y la exposición de los resultados de la investigación, también a través de los métodos y herramientas de comunicación más actualizados, se alcanza el desarrollo de formas de participación, de solidaridad y protección suscitadas por la innata necesidad de respeto y protección de los bienes examinados en una visión multidisciplinar de educación a la conciencia y responsabilidad y, por tanto, de educación para la ciudadanía.

En concreto, el concepto de "patrimonio» se destaca en varias ocasiones, en los documentos ministeriales (Indicaciones Nacionales de 2012 y en el Plan Nacional de Educación Patrimonial de 2019) en la necesidad de conocer el patrimonio circundante y la observación directa para así poder reconocer la sedimentación de civilizaciones legibles en las ciudades y en las huellas conservadas en el paisaje. Aspectos que involucran todas las clases sociales y disciplinas, encaminadas a identificar problemas relacionados con la protección y valorización del patrimonio natural y cultural, proponiendo soluciones adecuadas en el contexto de la vida del niño y la niña. Las actividades vivenciales están orientadas a adquirir una conciencia histórica que sea capaz de motivar y asumir un sentido de responsabilidad hacia el patrimonio y los bienes comunes, promoviendo acciones de cuidado y mejora, como

\footnotetext{
41 Ibidem.
} 
la salvaguarda, recuperación y conservación del propio patrimonio (Indicaciones Nacionales, 2012, p. 73).

La realización de estas experiencias se convierte, por lo tanto, en la culminación natural de la investigación, realizada tanto sobre el patrimonio cultural local como sobre el más amplio y global, precisamente en función de la relación directa antes mencionada, entre los niveles de conocimiento de la Historia que generó este patrimonio, y las formas de respeto y protección que asumen hacia él. Si estas prácticas didácticas pueden considerarse satisfactorias en los contextos del Jardín de Infancia y la Escuela Primaria (con experiencias vinculadas a contextos de la Historia reciente y por lo tanto de la Historia local), a menudo son difíciles de implementar en los grados superiores, como atestiguan las consideraciones siguientes, a propósito de la profesionalización docente y las adquisiciones de investigaciones recientes realizadas sobre el tema.

La educación patrimonial también se lleva a cabo con la participación activa por décadas de iniciativas, que se desarrollan en el territorio nacional y que consisten en el reconocimiento de los valores del territorio en el que viven los estudiantes, así como la puesta en marcha de talleres orientados a la atención individual y en comunidades del patrimonio cultural (Branchesi, lacono y Reggio, 2020). Entre ellos tenemos: los días europeos del patrimonio, la iniciativa nacional de "adoptar un monumento", la ciudad de los niños (Tonucci, 1997), "los parlamentos de los estudiantes" (Borghi y Dondarini, 2015) y muchos otros eventos locales activados en colaboración con instituciones, asociaciones, museos, bibliotecas, archivos y escuelas. Iniciativas fundamentales para evitar el riesgo de afrontar un futuro con fronteras no bien definidas, sin ni siquiera conocer adecuadamente las premisas y raíces de los temas más inquietantes de la actualidad, como el de la convivencia y la legalidad, el de los límites y desequilibrios del desarrollo y el respeto al entorno.

\subsection{Conocimiento del patrimonio y formación del profesorado, entre la investigación y la formación}

En los lugares de formación docente es donde se deben abordar las cuestiones básicas de la educación, para no dejar a los futuros profesores enfrentados solos y sin preparación al muy delicado problema de los métodos, el cumplimiento y la eficacia de su enseñanza. Es fundamental proponer itinerarios derivados de experiencias reales en los que se ha buscado una convergencia efectiva de investigación y docencia. Los cursos de formación específicos deben estar orientados a la adquisición de competencias y contenidos en un cuadro de conocimientos, que privilegie los aspectos metodológicos. En definitiva, tanto para la Historia como para otras asignaturas, las metodologías y los problemas de enseñanza no pueden quedar relegados a un segundo plano, sin embargo, casi siempre son excluidos del currículo 
normal de los futuros profesores y la «didáctica» debe abordarse con atención y un cuidado no inferior a la disciplina maestra.

Para formar docentes es necesario saber motivarlos, interesarlos y estimularlos y, para ello, es necesario dotarlos de una seria preparación disciplinaria, pedagógica, metodológica, y dotarles de un conocimiento del entorno escolar y de las distintas instituciones de formación que operan en la zona. La conexión UniversidadEscuela-Territorio es de vital importancia y la esencialidad de esta unión es el eje sobre el que basar la formación del futuro docente, que, por tanto, deberá dominar competencias disciplinarias específicas, metodologías de investigación y saber combinar estos conocimientos en la didáctica práctica.

Para una formación de calidad es necesario un diálogo compartido, activo y participativo entre los niveles institucionales y territoriales y que los distintos temas se reflexionen de forma conjunta en los cursos de formación, entre la difusión de conocimientos y las habilidades operativas. Por lo tanto, la Universidad juega un papel fundamental en la construcción de un proceso continuo de formación entre la preparación inicial de los docentes, su especialización, el ejercicio de su profesión y la actualización constante.

Precisamente en relación con los aspectos ambientales y territoriales, hay que señalar que en la bibliografía generada a menudo persiste un enfoque rígidamente dualista que contrasta naturaleza y hombre, espontáneo y artificial, natural e histórico. Fueron las ciencias biológicas y físicas las que sentaron las bases para una lectura no dicotómica entre el hombre y el «mundo exterior». En esta nueva armonía, incluso el conocimiento histórico puede contribuir a una correcta educación ambiental. El medio ambiente, el territorio, el paisaje, como resultado actual de procesos milenarios de transformación, son temas sobre los que construir nociones básicas y comunes y elaboraciones subjetivas, en ese diálogo circular entre lo local y lo general, necesario para un respeto activo de ambos microsistemas y de todo el ecosistema.

La educación para la sostenibilidad, como se lee en los documentos de la Década de la Educación para el Desarrollo Sostenible (DESS-UNESCO) 2005-2014, proclamada por la Asamblea General de la ONU, hace referencia a una educación en valores, en cuyo centro se encuentra el respeto por los demás, por la diferencia y la diversidad, por el medio ambiente, por los recursos de la tierra. El desafío de la revolución green requiere la formación de personas con habilidades críticas, respetuosas de la complejidad de la realidad y capaces de empezar por las cosas simples, por las relaciones, de cooperar, para tejer un diálogo fructífero e innovador con los demás. Garantes de un sano y cordial vivir mundial. La educación para la sostenibilidad tiene como referencia constante el desarrollo de una identidad personal a la par que comunitaria, debe propiciar la creación de situaciones de colaboración para así experimentar formas de participación activa en la vida del propio territorio, facilitando el encuentro dialógico entre generaciones y culturas. El conocimiento y 
conservación de los testimonios del pasado y la renovación de la memoria deben interactuar con la apertura hacia el futuro, a través del ejercicio de la imaginación y la planificación común. En este sentido, toda práctica educativa debe ser a su vez responsable y verificada constantemente, porque si se quiere promover en las personas la capacidad de considerar las consecuencias a largo plazo, de sus acciones, de pensar de manera transversal en un mundo en el que el conocimiento de la realidad y de nosotros mismos está cambiando constantemente, cada práctica «... no es un destino [...] sino una forma de viajar» (Scott y Grough, 2003).

Una reciente encuesta (Galletti, 2020) en la que participaron estudiantes del Máster de Educación Primaria de ciclo único de la Università di Bologna, atestiguó que la práctica docente de la enseñanza de la Historia sigue estando fuertemente vinculada (la referencia es a las Escuelas Secundarias de I y II grado) al modelo tradicional de enseñanza unidireccional, que se ancla a la presentación de hechos históricos por parte del docente y la consecuente memorización de los mismos por parte de los estudiantes con poca atención a la formación de un sentido crítico. Sin embargo, debemos aclarar, como ha sido ampliamente comentado por estudios europeos (Estepa-Giménez, 2017), y por los recientes eventos de salud epidémica global de Covid-19, que el uso y desarrollo de nuevas tecnologías para apoyar la práctica docente ha sido fundamental para dar una respuesta inmediata a la emergencia, pero también plantearon temas y reflexiones sobre el uso y efectividad de las nuevas tecnologías, sin duda una reflexión sobre la enseñanza de la misma Historia. La experiencia de la pandemia ha puesto en evidencia cuán probablemente fuera el hecho que la docencia de la disciplina no quisiera abordar, si no casualmente en la última década la falta de adaptabilidad de la escuela, y en particular la referencia es al aprendizaje y la enseñanza de la asignatura Historia, a las necesidades de la sociedad y por tanto de los niños y niñas. Por lo tanto, hay que señalar que la educación patrimonial está presente de manera esporádica y escasa en los currículos escolares de los centros de Secundaria inferior y en la propia práctica docente; esto debe estar vinculado a una insuficiente formación universitaria de los futuros profesores (Borghi y Cuenca López, 2003; Galletti, 2020). Por otra parte, existe una visión de la enseñanza de la disciplina que debe presuponer el conocimiento mismo del significado de "patrimonio» (a menudo una visión limitada por parte de los profesores, que se orienta casi exclusivamente a elementos monumentales únicamente, dejando de lado por ejemplo los aspectos del patrimonio inmaterial y por lo tanto una visión holística) y la falta de adquisición de competencias históricas y sociales en una perspectiva inter y multidisciplinar (Prats Cuevas, 2001).

Regresando por lo tanto a los resultados de la encuesta mencionada anteriormente, confirmamos -nuevamente- la brecha entre los anhelos y los logros reales de la docencia, debido a las dificultades diarias que tienen que afrontar los docentes, entre las que resaltan, en primer lugar, la carga docente y un programa enorme y complejo que deja poco espacio, especialmente para los grados superiores, para 
una enseñanza eficaz integrada con otras materias como antropología, arte, derecho, filosofía, literatura, música, ciencias, tecnologías, Historia y Geografía.

\subsection{Las habilidades. Los temas y enfoques}

Entre los numerosos documentos elaborados por la Unión Europea, destinados a proveer de uniformidad pedagógica básica al sistema escolar europeo, la Recomendación del Parlamento Europeo y del Consejo del 18 de diciembre de 2006 identifica ocho competencias clave para el aprendizaje permanente, destinadas a la adquisición de los conocimientos necesarios para que cada ciudadano pueda integrarse con éxito en el entorno social y laboral. Las competencias clave se refieren a la «combinación de conocimientos, habilidades y actitudes adecuadas para hacer frente a una situación particular» (en «La Recomendación sobre competencias clave»), que por lo tanto nos permite responder a los cambios constantes en la sociedad, e identificados en:

1. Comunicación en la lengua materna.

2. Comunicación en lenguas extranjeras.

3. Competencia matemática y competencias básicas en ciencia y tecnología.

4. Competencia digital.

5. Aprender a aprender.

6. Competencia social y cívica.

7. Espíritu de iniciativa y espíritu empresarial.

8. Conciencia y expresión cultural.

Con respecto a las competencias clave antes mencionadas (Audigier, 2002, pp. 56-183), se recomienda que el diseño de unidades de aprendizaje y laboratorios de Historia esté fuertemente orientado a la formación de habilidades y conocimientos en la perspectiva de competencias, que conciernen en particular la competencia digital, aprender a aprender, la competencia social y cívica y la conciencia y expresión cultural (Borghi, 2014). Habilidades consideradas esenciales para la realización y el desarrollo de las bases para el aprendizaje permanente y el desarrollo personal en una perspectiva de ciudadanía activa e inclusión social, que si, por una parte, no renuncian a los contenidos de la disciplina Historia, por otra, centran la atención en las actitudes del alumno, en sus motivaciones personales, en su pensamiento crítico hacia lo que le rodea, aprendizaje que lo acompañará a lo largo de su vida (Borghi et al., 2015).

Por lo tanto, en nuestro discurso encontramos que en los últimos 20 años ha habido varias reformas, incluida la reforma Moratti de 2003, la reforma Gelmini de 2008 y la Buena Escuela de 2015, que han traído cambios importantes, algunos de los cuales aún están presentes hoy. La referencia es con respecto a la abolición 
del examen estatal en el quinto año de la Escuela Primaria, alternancia obligatoria escuela-trabajo en cualquier escuela Secundaria y formación obligatoria y permanente del profesorado en servicio. Sin embargo, no faltan actualizaciones y en 2019 asistimos a la aprobación del texto de la ley sobre la introducción de la educación cívica "como asignatura separada e incluida en el plan de estudios de Primaria y Secundaria ${ }^{42}$. A partir del 1 de septiembre del curso académico 2020/2021, de hecho, en el primer ciclo de educación, con la Ley 92/2019, se estableció la docencia transversal de educación cívica, por 33 horas al año, para cada año del curso. La innovación es, sin duda, atribuible a la transversalidad de la asignatura que, superando las limitaciones de la disciplina, debería garantizar un enfoque holístico, global, multiperspectívico así como el desarrollo de los procesos de aprendizaje. De hecho, la identificación de un conjunto de temas que las líneas de formación recientes consideran imprescindibles, se orienta a la formación integral del ciudadano.

En este sentido, la adquisición de las habilidades ciudadanas clave que son ciertamente individuales se ve alimentada por la conciencia y la mejora de la dimensión relacional, social y emocional del aprendizaje. En particular, los temas de referencia se refieren a cada orden y grado:

1. Constitución, institución del Estado italiano, la Unión Europea y organizaciones internacionales; Historia de la bandera y el himno nacional.

2. Agenda 2030 para el Desarrollo Sostenible.

3. Educación de ciudadanía digital.

4. Elementos fundamentales del derecho, con especial atención al derecho laboral.

5. Educación ambiental, desarrollo sostenible.

6. Educación para la legalidad y lucha contra las mafias.

7. Educación para respetar y mejorar el patrimonio cultural y los bienes públicos comunes.

8. Formación básica en protección civil.

9. Educación vial.

10. Educación para la salud y el bienestar.

11. Educación para el voluntariado.

La ley de la nueva Educación Cívica tiene la ventaja de identificar un conjunto de temas que los lineamientos de formación más recientes ahora consideran imprescindibles. Esta es una tendencia interesante, que gira en torno a la promoción del pensamiento crítico, la creatividad, de la conciencia social y cultural como herramientas preciosas para combatir todas las formas de violencia, injusticia y discriminación. Además, las indicaciones combinan las necesidades del mercado laboral y el progreso económico con la salvaguarda de la sensibilidad ética a los temas sociales,

42 Italia: Reformas en la educación escolar, Red EURYDICE. https://eacea.ec.europa.eu/ national-policies/eurydice/content/national-reforms-school-education-33_it 
de acuerdo con los objetivos de la Agenda 2030 de la ONU para un desarrollo sostenible, equitativo e inclusivo, que también incluye el objetivo de una calidad educación, igualmente equitativa e inclusiva.

Con respecto a los contenidos históricos de los programas ministeriales mencionados anteriormente, para la escuela Secundaria inferior (desde la antigüedad tardía hasta el humanismo y el Renacimiento - clase I; desde los descubrimientos geográficos y la expansión europea hasta la Restauración y el Risorgimento - clase II; desde la sociedad de masas hasta el final del bipolarismo y los problemas del mundo de hoy - clase III), la educación cívica está vinculada a temáticas históricas a partir del conocimiento de la Constitución (Dignidad y Derechos, 1. a clase); desde el análisis de la evolución de algunos conceptos culturales en la Historia y cómo se manifiestan en diferentes contextos sociales, desde las formas del arte en el tiempo y el espacio, hasta la Historia de las religiones (diálogo intercultural, clase II); del medio ambiente y las intervenciones humanas a lo largo de la Historia, en particular las consecuencias ambientales y económicas a escala local y global de las huellas humanas (desde la inestabilidad hidrogeológica, los fenómenos meteorológicos extremos, la desertificación, la contaminación, el sobrecalentamiento, etc.); profundizar en la clase III el orden de la República, la organización política y económica de la UE, la Carta de Derechos de la UE y la Constitución Europea y organismos internacionales (ONU, Unesco, Tribunal Internacional de La Haya, Alianza Atlántica, Unicef, Amnistía Internacional, Cruz Roja). Los contenidos y actividades son transversales en todas las disciplinas y están conectados con las competencias clave sociales y cívicas europeas. Del total de 33 horas de Educación Cívica que se deben realizar en cada clase por año, el desglose de las horas semanales realizadas para cada disciplina es indicativo (esta es una propuesta adoptada en principio, siempre considerando que hay autonomía escolar) tal como se muestra a continuación:

- Letras (Italiano, Historia, Geografía, donde solo hay un profesor): 11.

- Matemáticas y Ciencia: 7.

- Idioma inglés: 4.

- $\quad$ Arte: 2.

- Tecnología: 2.

- Música: 2.

- $\quad$ Ed. Física: 2.

- Religión / alternativa: 1.

Partiendo también del análisis de los libros de texto, se evidencia la necesidad de estimular a los estudiantes en caminos/experiencias vivenciales, orientados a la construcción del pensamiento crítico, que en lo que a Historia se refiere tiene como objetivo saber interpretar las fuentes de manera crítica, evaluando la confiabilidad (pensar en el enfoque de la ciudadanía digital), en la capacidad de poder 
argumentar los conocimientos y conceptos aprendidos utilizando el lenguaje específico de la disciplina y ser capaz de distinguir entre desarrollo histórico, microrrelatos e Historias sectoriales o temáticas, con la finalidad de comprender las relaciones entre la Historia local, la Historia nacional o mundial. Es evidente el papel fundamental que asume la comunicación, para lo cual es necesario adquirir habilidades operativas congruentes con la actual "sociedad de la información», salvaguardando los aspectos narrativos que siempre han caracterizado la difusión de la Historia y aprovechando las extraordinarias oportunidades que presentan las nuevas tecnologías, aunque con todas las advertencias y precauciones inducidas por la facilidad de manipulación y distorsión de la información. Por lo tanto, es evidente que la docencia tiene como objetivo formar ciudadanos conscientes, capaces de desarrollar el pensamiento crítico, así como una acción autónoma y responsable dentro de la sociedad. La participación en la vida de la propia comunidad es fundamental a través de la potenciación de las relaciones interpersonales y entre las instituciones, centros de agregación cultural, familias y territorio. La evaluación, con las nuevas disposiciones del año escolar en curso (2020/2021), prevé juicios descriptivos en lugar de las notas en décimas que estaban en vigor desde 2008 con la reforma Gelmini.

Con respecto a la educación cívica en las Escuelas Secundarias (14-19 años), cabe señalar que la docencia es impartida por docentes capacitados en la enseñanza de las disciplinas jurídicas y económicas, si están disponibles en la autonomía; aunque hay que precisar que no se debe descuidar la dimensión transversal de la disciplina, cuyos temas no pueden remontarse únicamente al Derecho. Por tanto, la docencia está organizada de tal forma que se abordan cuestiones no estrictamente relacionadas con las disciplinas jurídico-económicas mencionadas.

En el contexto de la autonomía escolar, las escuelas pueden promover experiencias extracurriculares para integrar y/o potenciar la enseñanza de la educación cívica mediante el establecimiento de redes, incluso de duración plurianual, con otras asignaturas institucionales, con el mundo del voluntariado y con el Tercer sector y con los municipios (sobre el conocimiento del funcionamiento de las administraciones locales y sus órganos, conocimiento histórico del territorio y uso estable de los espacios verdes y espacios culturales, conocimiento del funcionamiento de las administraciones locales). Observando y analizando los programas de Historia de las Escuelas Secundarias superiores y las conexiones con la educación cívica, podemos ver que cada núcleo temático abordado en los cinco años de escuela está orientado y apoyado por los propios manuales de la disciplina a vínculos interdisciplinarios con el Derecho (por ejemplo, a la diferencia entre los códigos de las civilizaciones antiguas y la comparación con los códigos modernos) a los conceptos de igualdad presentes en la legislación griega y el concepto de igualdad en las constituciones modernas; de los vínculos con el derecho romano a la Constitución italiana, con una reflexión sobre el concepto mismo de ciudadanía europea, encaminada en 
particular a saber distinguir los conceptos de hombre, ciudadano, persona, individuo, y entrelazando los mismos artículos con la experiencia personal del estudiante; rastrear situaciones de discriminación y marginación; estrechos vínculos con la Constitución italiana y la Carta Europea para enfoques generales que incluyen visiones del mundo y que conducen al derecho internacional. Con respecto al tema del poder temporal y religioso de la Iglesia, desde la Donación de Constantino a los Pactos de Letrán, la conexión con la educación cívica se expresa a través del conocimiento de las políticas de integración y el derecho al asilo; de los vínculos de los contenidos de la Historia (tolerancia religiosa, diálogo entre religiones, humanidad y guerra, la Constitución rechaza la guerra, la defensa del Estado y el papel del ejército y las organizaciones mundiales), que mediante métodos de laboratorio desentraña los orígenes históricos de relaciones internacionales. Nuevamente se reitera la importancia del respeto a la persona y el valor de las relaciones interpersonales e interinstitucionales entre los centros de agregación cultural y relacional de los jóvenes, las familias y el territorio.

El artículo 4 del Decreto Ministerial prevé medidas de formación, apoyo y seguimiento por parte del Ministerio de Educación para la primera fase de ejecución (2020/2022). La formación y el acompañamiento tendrán como destinatarios a los responsables de las escuelas y al profesorado; el seguimiento de lo que hacen las escuelas se implementará de acuerdo con los tiempos, formas y métodos definidos por el propio Ministerio de Educación.

\section{ALGUNAS CONSIDERACIONES FINALES. PERSISTENCIA Y HORIZONTES EN LA ENSEÑANZA DE LA HISTORIA Y LA FORMACIÓN DEL PROFESORADO}

En su continua y necesaria transición, las diversas materias de la formación escolar, cultural y científica se enfrentan a una fase de especial dificultad, ya que, ante las rápidas y completamente inéditas transformaciones de la actualidad, la desorientación, inercia, contradicciones y anacronismos se manifiestan de muchas maneras cada vez más evidentes. Particularmente sufren los maestros de todos los grados y disciplinas que solo parcialmente logran cumplir y, a veces, incluso reaccionan positivamente a los nuevos desafíos, pero que a menudo se sienten solos haciendo malabarismos entre regulaciones internas contradictorias y limitantes y expectativas externas, y recurrentemente en gran medida desactualizadas. En este contexto de dificultad generalizada, surge aún más claramente la subestimación de los aspectos didácticos por parte de los círculos académicos, en los que se ha consolidado una contradicción letal desde hace algún tiempo: una ostentación generalizada de apreciación por las necesidades de la docencia se contrarresta con un persistente e idéntico desprecio y/o descrédito sustancial y generalizado de todo lo didáctico e informativo. 
Así, tras la pantalla de las intenciones en regla, y rechazando el diálogo necesario con los verdaderos protagonistas y sujetos de aprendizaje, el propio espacio destinado a formar y actualizar al profesorado no está disponible ni preparado para una de sus principales funciones. Por el contrario, para hacer frente a los crecientes y alarmantes síntomas de inadecuación de sus cursos, los docentes universitarios deben apropiarse de la problemática docente, no solo para una formación docente rentable, sino también y sobre todo para sacar los frutos de esas experiencias, que tratan concretamente los delicados problemas tanto de métodos y contenidos, como de correspondencia y eficacia de la enseñanza en las diversas disciplinas.

Tanto para la Historia como para otras asignaturas, la atención a las estrategias didácticas acordes con las dificultades reales que los docentes encuentran a diario en sus oficinas no puede quedar eclipsada por la de los contenidos disciplinarios. Por lo tanto, la respectiva "didáctica» debe abordarse con atención y esmero, nunca inferior al que se dispensa a las disciplinas tradicionales (matrices), proponiendo cursos orientados a experimentar con los itinerarios y las herramientas fundamentales de la investigación y la docencia, en un marco de procesos formativos que privilegien lo metodológico y también ofrezcan las nociones y contenidos básicos para luego diseñar subjetivamente caminos didácticos coherentes y efectivos. En esta perspectiva también es posible encontrar un equilibrio efectivo en el contraste, a veces exagerado, entre quienes apoyan la importancia primordial de los contenidos y quienes, en cambio, otorgan mayor importancia a las metodologías.

También quisiéramos destacar (quizás lamentablemente) una cierta continuidad con respecto a las Indicaciones Nacionales. Respecto a estas últimas, ha habido una actualización con respecto a las de 2007 pero, de hecho, los principios constitutivos y aspectos disciplinarios en la base no han desaparecido. En el primer ciclo de educación «se asignan conocimientos históricos relacionados con el período desde la aparición del hombre hasta la antigüedad tardía» (Borghi, 2016, p. 120). Se promueve el conocimiento sobre la antigüedad tardía hasta principios del siglo XXI en la escuela Secundaria inferior; mientras que en la escuela Secundaria superior se promueve el conocimiento sobre la Historia antigua y medieval temprana hasta nuestros días. Este desglose curricular de la Historia no está exento de críticas (Dondarini, 2008). De hecho, la Historia medieval, moderna y contemporánea queda excluida del primer ciclo educativo, delegando el estudio de estas épocas al primer ciclo de Secundaria. Las consecuencias de esta decisión ministerial son diferentes. En primer lugar, no se ofrece la posibilidad (a los docentes de Primaria) de abordar temas de actualidad relacionados con los períodos históricos señalados anteriormente, ya que los estudiantes no tendrían los conocimientos al respecto. Al mismo tiempo, se alimenta el desconocimiento de los alumnos, porque están excluidos a priori de participar en proyectos educativos escolares y extraescolares relacionados con estos períodos históricos. Esto no solo significa excluirlos de cursos 
de formación interesantes, sino evitar que participen activamente en la vida de la ciudad y las oportunidades que ofrece.

En resumen, para que la enseñanza-aprendizaje de la Historia sea cada vez más significativa y acorde con esta rica orientación epistemológica, los aspectos sobre los que podemos seguir trabajando son diferentes.

Por ejemplo:

1. Formación de profesores. En particular, entre los docentes es donde precisamente se mantiene una subestimación del papel educativo de la asignatura, y a su vez, se forman en el ámbito académico con enfoques nocionales y meramente transmisores (salvo en casos excepcionales y, en particular, en la formación dirigida a futuros jardines de Infancia y educación infantil). Por otra parte, los maestros de Escuela Primaria no suelen estar capacitados desde el punto de vista didáctico, lo que no les permite disponer de herramientas para planificar caminos activos y significativos; sin apoyos particulares, continúan apoyando las lecciones en el uso de un manual, que a menudo es solo un resumen de los eventos más importantes, sin ninguna explicación de la conexión entre el pasado y el presente, y de hecho, a menudo, sin ninguna referencia a los eventos y periodos más actuales (el manual en sí no estimula la investigación y el estudio). La falta de actualización del profesorado, desde este punto de vista, alimenta el círculo vicioso que ve la Historia como una disciplina poco útil o, en todo caso, no indispensable.

2. La preparación de caminos verdaderamente significativos y motivadores. Ser capaz de programar módulos, unidades de aprendizaje, talleres, etc.; ser capaz de activar a los estudiantes y mejorar los elementos fundamentales de la disciplina (por ejemplo, el conocimiento del pasado y de las propias raíces; la mejora del patrimonio; ser capaz de sensibilizar sobre la protección y la participación en la comunidad; la capacidad de interpretar y obtener información de las fuentes; etc.). Estos caminos son capaces de desarrollar conocimientos, habilidades y llevar a los niños a las primeras formas de compartir y a la participación activa en la vida de la comunidad, no solo en un sentido local, sino también en un sentido global.

3. Un programa cada vez más capaz de relacionarse con el territorio y toda la comunidad educativa. Construir relaciones de colaboración con los distintos actores presentes en la zona (museos, asociaciones, archivos, etc.) para que la propuesta educativa sea siempre rica y "ubicada en el contexto», y para que el valor de estos recursos sea evidente también para los niños, con la esperanza de construir vínculos y relaciones que se prolonguen en el tiempo, con mira al aprendizaje permanente.

En conclusión, solo podemos señalar que en una sociedad cada vez más híbrida y compleja, también debe estar orientada a promover una formación cultural basada en la conciencia de las peculiaridades y diversidades de la convivencia, en el convencimiento de que toda identidad evoluciona y que cualquier presunción de su inmovilidad, es decir con el justificar el rechazo de las nuevas llegadas y los cambios 
relacionados, es infundada. Esto se puede comprobar en diversos campos a partir del socio-antropológico, ya que la necesidad de recuperar la adhesión a los patrimonios histórico-culturales de nuestras comunidades se ha visto acentuada por la formidable aceleración de las transformaciones en los modos de vida individuales y colectivos.

\section{REFERENCIAS BIBLIOGRÁFICAS:}

Aquilini, E., (2016). I programmi scolastici dell'Italia unita e le scienze: dal 1860 al 1955. Aracne Editrice.

Audigier. F. (2002). Concetti di base e competenze chiave per l'Educazione alla Cittadinanza Democratica. Scuola \& Cittá, (1), pp. 156-183. http://www. edscuola.it/archivio/antologia/scuolacitta/audigier.pdf

Ávila, R. M., Borghi, B., \& Mattozzi, I. (eds, 2009). L'educazione alla cittadinanza europea e la formazione degli insegnanti. Pátron editore.

Bonetta, G. (1977). Storia della scuola e delle istituzioni educative. Scuola e processi formativi in Italia dal XVIII al XX secolo. Giunti.

Borghi, B. (2008). Un patrimonio di esperienze sulla didattica del Patrimonio, Pátron editore.

Borghi, B. (eds. 2014). Educare alla cittadinanza attiva. Infanzia, (4/5), pp. 253259. https://www.rivistainfanzia.it/pvw/app/1PWDIN02/pvw_sito.php?sede_ codice=1PWDIN02\&page $=1916720$

Borghi, B. y Dondarini, R. (2015). Le radici per volare: Ricerche ed esperienze del Centro internazionale di Didattica della Storia e del Patrimonio. Minerva edizioni.

Borghi, B., García Pérez, F.F., \& Moreno-Fernández, O. (2015). Novi cives: cittadini dall'infanzia in poi. Pátron editore.

Branchesi, L. (2004). Il patrimonio culturale e la sua pedagogia per l'Europa. Armando Editore. 
Branchesi, L., lacono, M. R., \& Reggio, A. (2020). Educazione al Patrimonio Culturale in Italia e in Europa. Esperienze, modelli di riferimento, proposte per il futuro. Editore mediaGEO.

Bruni, E.M. (2005), Greco e latino: le lingue classiche nella scuola italiana, 18602005. Armando editore.

Cajani, L. (2019). I recenti programmi di storia per la scuola italiana. Storicamente: Laboratorio di storia, (15), pp. 1-42. https://doi.org/10.12977/stor798

Cuenca López, J. M. (2003). Análisis de concepciones sobre la enseñanza del patrimonio en la educación obligatoria, Enseñanza de las Ciencias Sociales, (2), pp. 37-45. https://www.raco.cat/index.php/EnsenanzaCS/article/view/126155

Estepa Giménez, J. (2017). Otra didáctica de la Historia para otra escuela (Lección Inaugural. Curso 2017-2018). Universidad de Huelva. http://www.ub.edu/ histodidactica/images/documentos/pdf/estepa\%20libro.pdf

Fiore, F. (2001). Rincorrere o resistere? Sulla crisi della storia e gli usi della storia. Passato e presente, (52), pp. 97-116. https://www.torrossa.com/it/resources/ an/2197561

Frabboni, F., Cerini, G., \& Spinosi, M. (2002). Come cambia la scuola Primaria, Tecnodid.

Galletti, F. (2020). Una ricerca con i futuri maestri: la percezione del patrimonio degli studenti universitari. Didattica della storia, (2), pp. 73-91. https://dsrivista. unibo.it/article/view/11882

Gelmi, O. (2019). Pedagogia e didattica delle scuole nuove nei programmi nazionali della scuola elementare italiana del 1945. Formazione, lavoro, persona, (10), pp. 1-17. https://forperlav.unibg.it/index.php/fpl/article/view/130

Gough, S., \& Scott, W. (2003). Sustainable development and learning: Framing the issues. Routledge. https://doi.org/10.4324/9780203464625

Martorana, V., Ambrosi, A. y Savarino, M. (2001, noviembre). Giuseppe Lombardo Radice y la escuela serena. Materiales para el estudio de la metodología de la comunicación formativa y disciplinas pedagógicas. http://www. dubladidattica. it/lomradice.html 
Panciera, W. (2016). Insegnare storia nella scuola Primaria e dell'infanzia. Carocci editore.

Panciera, W., \& Zannini, A. (2013). Didattica della storia. Manuale per la formazione degli insegnanti. Mondadori education.

Prats Cuevas, J. (2001), Enseñar Historia: Notas para una didáctica renovadora. Junta de Extremadura.

Sorzio, P. (2011), Apprendimento e istituzioni educative: storia, contesti, soggetti. Carocci editore.

Tonucci, F. (1997). La verdadera reforma empieza a los tres años. Revista Investigación en la Escuela, (33), pp. 5-16. http://files.desarrollocompetenciasdiversidad. webnode.mx/200000036-4e05f4efeb/tonucci\%20la\%20verdadera\%20 reforma-2.pdf

Washburne, C. W. (1940), A Living Philosophy of Education. Trad. Goretti, M., \& Rombo, G. (1957), Una filosofia vivente dell'educazione. Le Monnier.

Zago, G. (2005). II dibattito sulla formazione degli insegnanti nella "Rivista pedagogica». Pensa Multimedia.

Zago, G. (2008). Da maestri a direttori didattici. Pensa Multimedia. 